\title{
ON THE EXISTENCE OF ASYMPTOTICALLY GOOD LINEAR CODES IN MINOR-CLOSED CLASSES
}

\author{
PETER NELSON, STEFAN H.M. VAN ZWAM
}

\begin{abstract}
Let $\mathcal{C}=\left(C_{1}, C_{2}, \ldots\right)$ be a sequence of codes such that each $C_{i}$ is a linear $\left[n_{i}, k_{i}, d_{i}\right]$-code over some fixed finite field $\mathbb{F}$, where $n_{i}$ is the length of the codewords, $k_{i}$ is the dimension, and $d_{i}$ is the minimum distance. We say that $\mathcal{C}$ is asymptotically good if, for some $\varepsilon>0$ and for all $i, n_{i} \geq i, k_{i} / n_{i} \geq \varepsilon$, and $d_{i} / n_{i} \geq \varepsilon$. Sequences of asymptotically good codes exist. We prove that if $\mathcal{C}$ is a class of $\operatorname{GF}\left(p^{n}\right)$-linear codes (where $p$ is prime and $n \geq 1$ ), closed under puncturing and shortening, and if $\mathcal{C}$ contains an asymptotically good sequence, then $\mathcal{C}$ must contain all $\mathrm{GF}(p)$-linear codes. Our proof relies on a powerful new result from matroid structure theory.
\end{abstract}

\section{INTRODUCTION}

For a finite field $\mathbb{F}$, let $\mathbb{F}_{\text {prime }}$ denote the unique subfield of $\mathbb{F}$ of prime order. For a linear code $C$, denote the length of $C$ by $n_{C}$, the dimension of $C$ by $k_{C}$, and the minimum Hamming distance of $C$ by $d_{C}$. In short, $C$ is an $\left[n_{C}, k_{C}, d_{C}\right]$ code. A class $\mathcal{C}$ of codes is asymptotically good if there exists $\varepsilon>0$ such that for every $n \in \mathbb{Z}^{+}$there is a code $C \in \mathcal{C}$ of length $n_{C} \geq n$ satisfying $k_{C} \geq \varepsilon n_{C}$ and $d_{C} \geq \varepsilon n_{C}$.

For every finite field $\mathbb{F}$, the class of linear codes over $\mathbb{F}$ is asymptotically good, as suitable random codes have nonvanishing rate and minimum distance. Our main result can be seen as a converse to this statement. Our result involves two standard operations on linear codes. Given a code $C$, the puncturing of $C$ at $i$ is the code obtained from $C$ by removing the $i$ th coordinate from each word. The shortening of $C$ at $i$ is the code obtained from $C$ by selecting only the codewords of $C$ having a 0 in position $i$, and then puncturing the resulting code at $i$.

Theorem 1.1. Let $\mathbb{F}$ be a finite field. If $\mathcal{C}$ is an asymptotically good class of linear codes over $\mathbb{F}$, then every linear code $C^{\prime}$ over $\mathbb{F}_{\text {prime }}$ can be obtained from a code $C \in \mathcal{C}$ by a sequence of puncturings and shortenings. 
In other words, the only asymptotically good classes of $\mathbb{F}$-linear codes that are closed under puncturings and shortenings are those that contain all linear codes over some field. This result was conjectured by Geelen, Gerards, and Whittle [3]. A restatement of the above theorem for prime fields is the following:

Theorem 1.2. Let $\mathbb{F}$ be a field of prime order. If $\mathcal{C}$ is a proper subclass of the linear codes over $\mathbb{F}$ that is closed under puncturing and shortening, then $\mathcal{C}$ is not asymptotically good.

When $|\mathbb{F}|=2$, this substantially generalises results of Kashyap [2] which show that the classes of graphic binary codes, as well as a slightly larger class of 'almost-graphic' codes, are not asymptotically good.

Our proof makes fundamental use of a deep theorem in structural matroid theory recently announced by Geelen, Gerards and Whittle. This theorem is one of many outcomes of the 'matroid minors project', the result of more than a decade of work generalising Robertson and Seymour's graph minors structure theorem [5] to matroids representable over finite fields. While this theorem has now been stated in print [3], its proof will stretch to hundreds of pages and has yet to be published. The reader should be aware of this contingency; for a more detailed discussion see 3 .

\section{PRELIMINARIES}

Since the ideas in our proof are matroidal, we adopt the terminology of matroid theory. The correspondence between linear codes and matroids is fairly direct even when a matroid is defined in the usual way by its ground set and rank function, but for convenience we will deal with a flavour of matroid whose definition coincides exactly with that of a linear code.

Represented matroids. If $\mathbb{F}$ is a field, then an $\mathbb{F}$-represented matroid is a pair $M=(E, U)$, where $E$ is a finite set and $U$ is a subspace of $\mathbb{F}^{E}$. We often omit ' $\mathbb{F}$-represented' when the context is clear. We write $|M|$ for $|E|$.

If $A$ is an $\mathbb{F}$-matrix with column set $E$ such that rowspace $(A)=U$, then we write $M=M(A)$; we call $A$ a generator matrix for $M$ and say that $A$ generates $M$. For $X \subseteq E$ we write $u \mid X$ for the restriction of the vector $u$ to those coordinates indexed by $X$, we write $U \mid X$ for the space $\{u \mid X: u \in U\}$, and we write $r_{M}(X)$ for the dimension of $U \mid X$. We denote $r_{M}(E)$ simply by $r(M)$. We call $r_{M}$ the rank function of $M$. 
If $C$ is a $q$-ary $[n, k, d]$ code, then $M=(\{1, \ldots, n\}, C)$ is a matroid with $|M|=n$ and $r(M)=k$. The rank function simply gives the dimension of this code and of all its puncturings.

If $A_{2}$ is an $\mathbb{F}$-matrix obtained from an $\mathbb{F}$-matrix $A_{1}$ by nonzero column scalings, then $M\left(A_{1}\right)$ and $M\left(A_{2}\right)$ are formally distinct but share the same rank function. We give a name to this equivalence: two $\mathbb{F}$ represented matroids $\left(E, U_{1}\right)$ and $\left(E, U_{2}\right)$ are projectively equivalent if $U_{2}=\left\{u D: u \in U_{1}\right\}$ for some nonsingular diagonal matrix $D$.

Matroid terminology. Matroid duality coincides with linear code duality. The dual matroid $M^{*}$ of an $\mathbb{F}$-represented matroid $M=(E, U)$ is defined to be $\left(E, U^{\perp}\right)$, where $U^{\perp}$ denotes the orthogonal complement of $U$. For $X \subseteq E$ we write $M \backslash X$ for $(E-X, U \mid(E-X))$ and $M / X$ for $\left(M^{*} \backslash X\right)^{*}$; these are the matroids obtained from $M$ by deletion and contraction of $X$ respectively; these operations correspond to puncturing and shortening of codes. If $N$ is an $\mathbb{F}$-represented matroid that is projectively equivalent to $M / C \backslash D$ for some disjoint subsets $C$ and $D$ of $E$, then we say $N$ is a minor of $M$. A class $\mathcal{M}$ of matroids is minor-closed if $\mathcal{M}$ is closed under taking minors and isomorphism.

A set $X \subseteq E$ is spanning in $M$ if $r_{M}(X)=r(M)$ and independent in $M$ if $r_{M}(X)=|X|$. If $X$ is not independent then $X$ is dependent. A circuit of $M$ is a minimal dependent set and a cocircuit of $M$ is a minimal dependent set of $M^{*}$, or equivalently a minimal set $C$ satisfying $r(M \backslash C)<r(M)$. For a matroid $M$, we write $g(M)$ for the size of a smallest circuit of $M$, also called the girth of $M$.

If $C$ is a $q$-ary $[n, k, d]$ code, then $M=(\{1, \ldots, n\}, C)$ is a matroid with $g\left(M^{*}\right)=d$. For that reason we sometimes write $d(M)$ for $g\left(M^{*}\right)$.

Connectivity. A notion fundamental in matroid theory that arises in our proof is that of connectivity. Informally, a matroid has low connectivity if it can be obtained by 'gluing' two smaller matroids together on a low-dimensional subspace. There are many notions of matroid connectivity but we just need one; for $t \in \mathbb{Z}^{+}$, a matroid $M=(E, U)$ is vertically $t$-connected if, for every partition $(A, B)$ of $E$ satisfying $r_{M}(A)+r_{M}(B)<r(M)+t-1$, either $A$ or $B$ is spanning in $M$. For instance, $M$ is vertically 2 -connected if and only if $M$ cannot be written as the direct sum of two positive-rank matroids. Note that other authors often use a more restrictive definition of "vertically $t$ connected," which implies ours.

Frame matroids. An $\mathbb{F}$-frame matrix is an $\mathbb{F}$-matrix in which every column has at most two nonzero entries, and an $\mathbb{F}$-represented frame matroid is a matroid having an $\mathbb{F}$-frame matrix as a generator matrix. 
For a group $\Gamma$, a $\Gamma$-labelled digraph is a pair $(G, \Sigma)$, where $G=(V, E)$ is a directed graph (allowing loops and multiple edges) and $\Sigma: E \rightarrow \Gamma$ is an assignment of a label in $\Gamma$ to every arc of $G$. There is a wellknown and natural correspondence between $\mathbb{F}$-frame matroids and $\mathbb{F}^{\times}$labelled digraphs; a full treatment is given in [4] and a reader familiar with these concepts can skip this subsection, where we just give the minimum definitions and observations we will need.

If $A$ is an $\mathbb{F}$-frame matrix with row set $V$ and column set $E$, then a graph representation of $A$ is an $\mathbb{F}^{\times}$-labelled digraph $(G, \Sigma)$, where $G=(V, E)$ and $(G, \Sigma)$ satisfies the following conditions:

- If $A[e]$ has two nonzero entries in rows $x$ and $y$, then $e$ is an arc of $G$ from $x$ to $y$ with label $-A[e, x] A[e, y]^{-1}$ or an arc of $G$ from $y$ to $x$ with label $-A[e, y] A[e, x]^{-1}$, and

- If $A[e]$ has exactly one nonzero entry, then $e$ is a loop of $G$ at $x$ with arbitrary label in $\mathbb{F}^{\times}-\{1\}$.

- If $A[e]=0$, then $e$ is a loop of $G$ with label 1 .

It is clear that one frame matrix may have many graph representations, and that graph representations always exist unless $|\mathbb{F}|=2$, where a frame matrix having a column with exactly one nonzero entry has no graph representations since $\mathbb{F}^{\times}-\{1\}$ is empty. However, appending a 'parity' row to a binary frame matrix yields another row-equivalent frame matrix where every column has even support. Since we can remove redundant rows from an arbitrary frame matrix to still have a frame matrix and append a single row in this way in the binary case, we have the following statement, which we apply freely.

Proposition 2.1. If $M$ is an $\mathbb{F}$-represented frame matroid, then there is a generator matrix $A$ of $M$ having a graph representation and at most $r(M)+1$ rows.

A cycle or path of a digraph $G$ will denote any cycle or path of the underlying undirected graph of $G$. Let $C$ be a cycle of $G$, and $v_{1}, e_{1}, v_{2}, e_{2}, \ldots, e_{k-1}, v_{k}=v_{1}$ be a corresponding alternating sequence of vertices and arcs of $G$ (there are two choices for this sequence). Let $C^{+}$be the set of $e_{i}$ such that $e_{i}$ is directed from $v_{i}$ to $v_{i+1}$ in $G$, and $C^{-}$be the set of all other $e_{i}$. The sign of $C$ (relative to this sequence) is $\prod_{e \in C^{+}} \Sigma(e) \prod_{e \in C^{-}} \Sigma(e)^{-1}$. We say that $C$ is a balanced cycle of $(G, \Sigma)$ if $\operatorname{sign}(C)=1$. Note that a cycle being balanced does not depend on the choice of sequence of vertices and edges. There is a well-known characterisation of the set of circuits of $M(A)$ in terms of the balanced and unbalanced cycles of $(G, \Sigma)$, but we will just use the following weaker statement, which is fairly straightforward to check by considering linear dependencies in the columns of $A[C]$. 
Proposition 2.2. If $(G, \Sigma)$ is a graph representation of an $\mathbb{F}$-frame matrix $A$ and $C$ is a balanced cycle of $(G, \Sigma)$ or a connected subgraph of $G$ of minimum degree 2 which is not a cycle of $G$, then $C$ is dependent in $M(A)$.

We say $\left(G, \Sigma^{\prime}\right)$ was obtained from $(G, \Sigma)$ by resigning if, for some $\gamma \in \Gamma$ and for some partition $(U, W)$ of the vertices of $G$, we have

$$
\Sigma^{\prime}(e)= \begin{cases}\gamma \Sigma(e) & \text { if } e \text { runs from } U \text { to } W \\ \gamma^{-1} \Sigma(e) & \text { if } e \text { runs from } W \text { to } U \\ \Sigma(e) & \text { otherwise. }\end{cases}
$$

It is easily checked that $(G, \Sigma)$ and $\left(G, \Sigma^{\prime}\right)$ have the same collection of balanced cycles. In the representation this corresponds to scaling the rows indexed by $W$ by a factor $\gamma$.

In what follows, we need to assume that the graph representation of a frame matroid is connected, which means that there is a path between every pair of vertices. It is easy to show that a vertically 3-connected matroid, with no elements $e$ such that $r_{M}(e)=0$, has the property that every pair of elements is in a circuit. A consequence of this is:

Lemma 2.3. Let $(G, \Sigma)$ be a graph representation of an $\mathbb{F}$-represented frame matroid $M$. If $M$ is vertically 3-connected and has no loops, then $G$ is connected.

Corollary 2.4. Let $M$ be an $\mathbb{F}$-represented frame matroid. If $M$ is vertically 3-connected, then $M$ has a graph representation $(G, \Sigma)$ with $G$ connected.

Asymptotically good matroids. Finally, we redefine asymptotic goodness, this time for matroids. For $\alpha, \beta \in \mathbb{R}$ we say a sequence $M_{0}, M_{1} \ldots$, of matroids is $(\alpha, \beta)$-good if $\left|M_{i}\right| \geq i, r\left(M_{i}\right) \geq \alpha\left|M_{i}\right|$ and $g\left(M_{i}^{*}\right) \geq \beta\left|M_{i}\right|$ for each $i \in \mathbb{Z}_{0}^{+}$. A class $\mathcal{M}$ of matroids is asymptotically good if $\mathcal{M}$ contains an $(\alpha, \beta)$-good sequence for some $\alpha, \beta \in \mathbb{R}^{+}$. Note that $\alpha, \beta \leq 1$ for any such $\alpha, \beta$. For any finite field $\mathbb{F}$, the class of all $\mathbb{F}$-represented matroids is such a class.

\section{Connectivity}

Our goal in this section is to show that, to prove our main result, it suffices to focus on highly connected matroids.

Lemma 3.1. If $t \in \mathbb{Z}$ and $\mathcal{M}$ is an asymptotically good minor-closed class of matroids, then the class of vertically t-connected matroids in $\mathcal{M}$ is asymptotically good. 
Proof. For each $\beta \in \mathbb{R}^{+}$let $A_{\beta}$ denote the set of all $\alpha \in \mathbb{R}^{+}$such that $\mathcal{M}$ contains an $(\alpha, \beta)$-good sequence. Let $B=\left\{\beta \in \mathbb{R}^{+}: A_{\beta} \neq \varnothing\right\}$. By assumption, $B$ is a nonempty interval with $\inf (B)=0$ and $\sup (B) \leq 1$; let $\beta_{\max }=\sup (B)$. Each nonempty $A_{x}$ is also such an interval; for each $x \in B$, let $\alpha_{\max }(x)=\sup \left(A_{x}\right)$, noting that $\alpha_{\max }(x) \leq 1$.

Let $\beta \in\left(\frac{2}{3} \beta_{\max }, \beta_{\max }\right)$ and let $\delta=\frac{\beta}{4(1-\beta)}$. Set $\alpha_{\max }=\alpha_{\max }(\beta)$ and let $\alpha \in\left(\left(1+\frac{1}{2} \delta\right)^{-1} \alpha_{\max }, \alpha_{\max }\right)$. We have $\beta \in B$ and $\alpha \in A_{\beta}$; let $M_{0}, M_{1}, \ldots$ be an $(\alpha, \beta)$-good sequence of matroids in $\mathcal{M}$. Since $2 \beta>\beta_{\max }$ we have $2 \beta \notin B$ so $A_{2 \beta}=\varnothing$ and in particular $\frac{1}{2} \alpha \notin A_{2 \beta}$. Moreover, we have $(1+\delta) \alpha>\alpha_{\max }$ so $(1+\delta) \alpha \notin A_{\beta}$. There are therefore integers $m_{1}$ and $m_{2}$ such that and every matroid $M \in \mathcal{M}$ with $|M| \geq m_{1}$ satisfies $g\left(M^{*}\right)<2 \beta|M|$ or $r(M)<\frac{1}{2} \alpha|M|$, and every matroid $M \in \mathcal{M}$ with $|M| \geq m_{2}$ satisfies $g\left(M^{*}\right)<\beta|M|$ or $r(M)<\alpha(1+\delta)|M|$. Let $m=\max \left(m_{1}, m_{2}, 8 t \alpha^{-1}\right)$.

3.1.1. For each $n \in \mathbb{Z}$ with $n \geq 2 \beta^{-1} m$, the matroid $M_{n}$ is vertically t-connected.

Proof. Suppose for a contradiction that $n \geq 2 \beta^{-1} m$ and $M=M_{n}$ is not vertically $t$-connected. Let $\left(X_{1}, X_{2}\right)$ be a partition of $E(M)$ with $r_{M}\left(X_{1}\right)+r_{M}\left(X_{2}\right)=r(M)+t^{\prime}-1$ with $t^{\prime}<t$ and $r_{M}\left(X_{1}\right), r_{M}\left(X_{2}\right) \geq t^{\prime}$. Let $N_{1}=M / X_{2}$ and $N_{2}=M / X_{1}$. Note that $N_{1}, N_{2} \in \mathcal{M}$ and that $r\left(N_{i}\right)=r(M)-r_{M}\left(X_{3-i}\right)>r(M)-t+1$, so $r\left(N_{1}\right)+r\left(N_{2}\right)>r(M)-2 t$. Since every cocircuit of $N_{1}$ or $N_{2}$ is a cocircuit of $M$, we have $g\left(M^{*}\right) \leq$ $\min \left(g\left(N_{1}^{*}\right), g\left(N_{2}^{*}\right)\right)$. We have

$$
\beta|M| \leq g\left(M^{*}\right) \leq \min \left(g\left(N_{1}^{*}\right), g\left(N_{2}^{*}\right)\right) \leq \frac{1}{2}\left(g\left(N_{1}^{*}\right)+g\left(N_{2}^{*}\right)\right)
$$

and, since $|M|=\left|N_{1}\right|+\left|N_{2}\right|$, we have either $g\left(N_{1}^{*}\right) \geq 2 \beta\left|N_{1}\right|$ or $g\left(N_{2}^{*}\right) \geq$ $2 \beta\left|N_{2}\right|$. We may assume that the first case holds. Since each of $X_{1}$ and $X_{2}$ contains a cocircuit of $M$, we have $\left|N_{i}\right|=\left|X_{i}\right| \geq g\left(M^{*}\right) \geq \beta|M| \geq$ $m$ for each $i \in\{1,2\}$. Since $\left|N_{1}\right|+\left|N_{2}\right|=|M|$, this implies that $\left|N_{1}\right| \geq \frac{\beta}{1-\beta}\left|N_{2}\right|$. Moreover, $m \geq m_{1}$ gives $r\left(N_{1}\right)<\frac{1}{2} \alpha\left|N_{1}\right|$. We have

$$
\begin{aligned}
\alpha|M| & \leq r(M) \\
& <r\left(N_{1}\right)+r\left(N_{2}\right)+2 t \\
& <\frac{1}{2} \alpha\left|N_{1}\right|+r\left(N_{2}\right)+2 t \\
& \leq \frac{3}{4} \alpha\left|N_{1}\right|+r\left(N_{2}\right),
\end{aligned}
$$

Where the last line uses $\frac{1}{4} \alpha\left|N_{1}\right| \geq \frac{1}{4} \alpha m \geq 2 t$. From this and $|M|=$ $\left|N_{1}\right|+\left|N_{2}\right|$ we get

$$
\begin{aligned}
r\left(N_{2}\right) & \geq \frac{1}{4} \alpha\left|N_{1}\right|+\alpha\left|N_{2}\right| \\
& =\alpha(1+\delta)\left|N_{2}\right|
\end{aligned}
$$


Now $\left|N_{2}\right| \geq m \geq m_{2}$ so $g\left(N_{2}^{*}\right)<\beta\left|N_{2}\right|$ by choice of $m_{2}$. However $g\left(M^{*}\right) \leq g\left(N_{2}^{*}\right)$ and $\beta\left|N_{2}\right|<\beta|M|$, so $g\left(M^{*}\right)<\beta|M|$, contradicting the definition of $M$.

By the claim, all but finitely many terms of the sequence $M_{0}, M_{1}, \ldots$ are vertically $t$-connected, so the class of vertically $t$-connected matroids in $\mathcal{M}$ is asymptotically good, as required.

\section{The Structure Theorem}

For each field $\mathbb{F}$ of prime characteristic $p$, we write $\mathbb{F}_{\text {prime }}$ for the unique subfield of $\mathbb{F}$ with $p$ elements. In this section we state the deep structural result on which our proof is based. Essentially the theorem states that for any minor-closed class $\mathcal{M}$ of $\mathbb{F}$-represented matroids not containing all $\mathbb{F}_{\text {prime-represented matroids, the highly connected }}$ members of $\mathcal{M}$ are 'close' to being an $\mathbb{F}$-represented frame matroid or its dual. We need to define our notion of distance.

Our distance metric is based on 'lifts' and 'projections'. If $M_{1}=$ $\left(E, U_{1}\right)$ and $M_{2}=\left(E, U_{2}\right)$ are $\mathbb{F}$-represented matroids and there is an F-represented matroid $M$ with ground set $E \cup\{e\}$ satisfying $M \backslash e=M_{1}$ and $M / e=M_{2}$, then we say that $M_{2}$ is an elementary projection of $M_{1}$ and $M_{1}$ is an elementary lift of $M_{2}$. For arbitrary $\mathbb{F}$-represented matroids $M_{1}$ and $M_{2}$ on a common ground set $E$, we write $\operatorname{dist}\left(M_{1}, M_{2}\right)$ for the minimum number of elementary lifts/projections required to transform $M_{1}$ into $M_{2}$. (It is clear that any matroid on $E$ can be transformed into the rank- 0 matroid on $E$ by a finite sequence of projections, so this distance is always finite.) It is easy to see that, if $\operatorname{dist}\left(M_{1}, M_{2}\right) \leq k$, then there is a matroid $M$ with ground set $E \cup C \cup D$ satisfying $M \backslash D / C=M_{1}$ and $M / D \backslash C=M_{2}$, where $|C|+|D| \leq k$. Each elementary lift and projection can change the rank of a subset by at most one. From this we can deduce the following lemma.

Lemma 4.1. Let $M, N$ be $\mathbb{F}$-represented matroids with $\operatorname{dist}(M, N) \leq k$. If $M$ is vertically $t$-connected, then $N$ is vertically $(t-2 k)$-connected.

The structure theorem, a weakened combination of Lemma 2.1 and Theorems 3.2 and 3.3 in [3], can now be stated.

Theorem 4.2. Let $\mathbb{F}$ be a finite field and let $\mathcal{M}$ be a minor-closed class of $\mathbb{F}$-represented matroids not containing all projective geometries

over $\mathbb{F}_{\text {prime. }}$ There exists $k \in \mathbb{Z}^{+}$such that every vertically $k$-connected matroid $M$ in $\mathcal{M}$ satisfies $\operatorname{dist}(M, N) \leq k$ or $\operatorname{dist}\left(M^{*}, N\right) \leq k$ for some $\mathbb{F}$-represented frame matroid $N$. 


\section{Small Circuits}

In this section we show that if $M$ is a rank- $r$ matroid with has significantly more than $r$ elements and $M$ or $M^{*}$ is close to a frame matroid or its dual, then the girth of $M$ is at most logarithmic in $r$. The primal case is slightly more difficult and will result from the following corollary of a result of Alon, Hoory and Linial [1] observed by Kashyap [2]:

Lemma 5.1. If $G$ is a graph with girth $g$ and average degree $\delta>2$, then $g \leq 4+\frac{\log (|V(G)|)}{\log (\delta-1)}$.

The next three results extend the above lemma to matroids that are close to frame matroids. As before, a cycle of a graph refers strictly to a set of its edges.

Lemma 5.2. Let $t \in \mathbb{Z}^{+}$and $\beta \in \mathbb{R}^{+}$. If $G$ is a graph with $|V(G)|=$ $n \geq \max \left(\left(\frac{4 t}{\beta \log (1+\beta)}\right)^{2},(1+\beta) e^{4}\right)$ and $|E(G)| \geq(1+\beta) n$, then $G$ has a collection of $t$ pairwise edge-disjoint cycles, each of size at most $\frac{2 \log n}{\log (1+\beta)}$.

Proof. Let $m=|E(G)|$ and $\alpha=2(\log (1+\beta))^{-1}$. By choice of $n$ we have $\frac{t \alpha \log n}{n}<\frac{t \alpha}{\sqrt{n}}<\frac{1}{2} \beta$. Let $\mathcal{C}$ be a maximal collection of pairwise disjoint cycles of $G$ such that $|E(C)| \leq \alpha \log n$ for each $C \in \mathcal{C}$. Assume for a contradiction that $|\mathcal{C}|<t$; let $G^{\prime}=G \backslash \cup \mathcal{C}$. We have $\frac{\left|E\left(G^{\prime}\right)\right|}{n} \geq$ $\frac{m-t \alpha \log (n)}{n} \geq 1+\beta-\frac{t \alpha \log (n)}{n}>1+\frac{1}{2} \beta$, so the average degree $\delta^{\prime}$ of $G^{\prime}$ is at least $2+\beta$. By maximality of $\mathcal{C}$, the graph $G^{\prime}$ has girth $g^{\prime}>\alpha \log (n)$, so Lemma 5.1 gives

$$
\alpha \log (n)<4+\frac{\log (n)}{\log \left(\delta^{\prime}-1\right)} \leq 4+\frac{\log (n)}{\log (1+\beta)}
$$

Rearranging gives $\log (n)<4 \log (1+\beta)$, contradicting $n \geq(1+\beta) e^{4}$.

Corollary 5.3. Let $\mathbb{F}$ be a finite field of order $q$, let $\beta \in \mathbb{R}^{+}$and $t \in \mathbb{Z}^{+}$. Let $M$ be an $\mathbb{F}$-represented frame matroid with graph representation $(G, \Sigma)$, such that $G$ is connected. If $M$ satisfies

$$
r(M) \geq \max \left(|\mathbb{F}|,(2 q-3) \beta^{-1}+1-q, \frac{1}{q-1}\left(\frac{4 t}{\beta \log (1+\beta)}\right)^{2}, \frac{1+\beta}{q-1} e^{4}\right)
$$

and $|M| \geq(1+q \beta) r(M)$, then there is a set $X \subseteq E(M)$ such that $r_{M}(X) \leq|X|-t$ and $|X| \leq \frac{4 t \log r(M)}{\log (1+\beta)}$.

Proof. Let $A$ be an $\mathbb{F}$-frame matrix generating $M$ and let $(G, \Sigma)$ be a graph representation of $A$. Pick a spanning tree $T$ of $G$. By repeatedly 
resigning, we may assume that the edges of $T$ have sign 1 . Note that $|E(T)| \geq r(M)-1$.

Let $G^{+}$denote the undirected graph with vertex set $V(G) \times \mathbb{F}^{\times}$and edge set

$$
\begin{aligned}
& \left\{\left(\left(\delta^{-}(e), \gamma\right),\left(\delta^{+}(e), \gamma\right)\right): e \in T, \gamma \in \mathbb{F}\right\} \\
\cup & \left\{\left(\left(\delta^{-}(e), 1\right),\left(\delta^{+}(e), \operatorname{sign}(e)\right)\right): e \in E(G) \backslash T\right\} ;
\end{aligned}
$$

that is, we take $q-1$ copies of $T$, and each directed edge $(u, v)$ not in $T$ with sign $\gamma$ connects the copy of $T$ corresponding to 1 with the copy of $T$ corresponding to $\gamma$. It is easy to check that each cycle of $G^{+}$, by projection onto the first coordinate, corresponds to either a balanced cycle of $(G, \Sigma)$, or a subgraph of $G$ of minimum degree 2 that is not a cycle. It follows from Proposition 2.2 that every cycle of $G^{+}$is dependent in $M$. Now,

$$
\begin{aligned}
\left|E\left(G^{+}\right)\right| & =(q-1)|E(T)|+|M|-|E(T)| \geq(q-2)(r(M)-1)+|M| \\
& \geq(q-1)(1+\beta) r(M)+\beta r(M)-(q-2) \\
& \geq(1+\beta)\left|V\left(G^{+}\right)\right|-(q-1)(1+\beta)+\beta r(M)-q+2 \\
& \geq(1+\beta)\left|V\left(G^{+}\right)\right|,
\end{aligned}
$$

where we use $|E(T)| \geq r(M)-1$ first, $|M| \geq(1+q \beta) r(M)$ second, $\left|V\left(G^{+}\right)\right| \leq(q-1)(r(M)+1)$ third, and $r(M) \geq(2 q-3) \beta^{-1}+1-q$ last. By our lower bounds for $n=|V(G+)| \geq(q-1) r(M)$ and Lemma 5.2, the graph $G^{+}$contains $t$ pairwise disjoint cycles, each of size at most $\frac{2 \log n}{\log (1+\beta)} \leq \frac{4 \log r(M)}{\log (1+\beta)}$. Each of these cycles of $G$ is dependent in $M$, and it is thus easy to see that their union satisfies the result.

Lemma 5.4. Let $k \in \mathbb{Z}^{+}, \beta \in \mathbb{R}^{+}$, and $\mathbb{F}$ be a finite field. There exists $c \in \mathbb{Z}$ such that if $M$ is a rank-r $\mathbb{F}$-represented matroid with $r>2$ and $|M| \geq(1+\beta) r$ and there is an $\mathbb{F}$-represented, connected frame matroid $N$ satisfying $\operatorname{dist}(M, N) \leq k$, then $g(M) \leq c \log r$.

Proof. Let $\beta^{\prime}=\frac{1}{2} \beta$ and $c \geq \max \left((2 q-3) \beta^{-1}+1-q,|\mathbb{F}|,\left(\frac{4(k+1)}{\beta^{\prime} \log \left(1+\beta^{\prime}\right)}\right)^{2}\right.$, $\left.\left(1+\beta^{\prime}\right) e^{4}\right)+k$ be an integer so that $4(k+1) \log (x+k) \leq c \log \left(1+\beta^{\prime}\right) \log x$ for all $x \geq c$. Since $\beta^{\prime}>0$ we know that $M$ has a circuit, so $g(M) \leq$ $r+1$. If $r \leq c-1$ then $g(M) \leq r+1 \leq c \leq c \log r$, so the result holds; we may thus assume that $r \geq c$.

Let $M^{+}$be a matroid and $C, D \subseteq E\left(M^{+}\right)$be sets of size at most $k$ so that $M^{+} / C \backslash D=M$ and $M^{+} / D \backslash C=N$. Since each $Y \subseteq E(M)$ satisfies $r_{M^{+}}(Y) \geq r_{M}(Y) \geq r_{M^{+}}(Y)-r_{M^{+}}(C) \geq r_{M^{+}}(Y)-k$ and a similar statement holds for $N$, we have $\left|r_{M}(Y)-r_{N}(Y)\right| \leq k$ for 
each $Y \subseteq E(M)$. In particular, $r+k \geq r(N) \geq r-k \geq c-k$. By choice of $c$, Corollary 5.3 implies there is a set $X \subseteq E(N)$ with $r_{N}(X) \leq|X|-(k+1)$ and $|X| \leq \frac{4(k+1) \log (r(N))}{\log \left(1+\beta^{\prime}\right)} \leq \frac{4(k+1) \log (r+k)}{\log \left(1+\beta^{\prime}\right)} \leq c \log r$. Now $r_{M}(X) \leq r_{N}(X)+k<|X|$, so $X$ contains a circuit of $M$ and therefore $g(M) \leq c \log r$, as required.

We now deal with the case when $M$ is close to the dual of a frame matroid. Here we show that the girth is bounded above by a constant.

Lemma 5.5. Let $k \in \mathbb{Z}^{+}, \beta \in \mathbb{R}^{+}$, and $\mathbb{F}$ be a finite field. There exists $c \in \mathbb{Z}$ so that, if $M$ is a nonempty $\mathbb{F}$-represented matroid such that $|M| \geq(1+\beta) r(M)$ and there is an $\mathbb{F}$-represented frame matroid $N$ with $\operatorname{dist}\left(M^{*}, N\right) \leq k$ and connected graph, then $g(M) \leq c$.

Proof. We may assume that $\beta \leq 1$. Let $c=\left\lceil 12 \beta^{-1}(3 k+1)\right\rceil$. If $|M| \leq c$ then the result clearly holds, so we will assume otherwise. Let $M^{+}$be a matroid and $C, D \subseteq E\left(M^{+}\right)$be sets of size at most $k$ so that $M^{+} / C \backslash$ $D=M^{*}$ and $M^{+} / D \backslash C=N$. As before, we have $\left|r_{M^{*}}(Y)-r_{N}(Y)\right| \leq k$ for each $Y \subseteq E\left(M^{*}\right)$. Let $A$ be an $\mathbb{F}$-frame matrix generating $N$ with $r(N)$ rows (or $r(N)+1$ rows if $|\mathbb{F}|=2)$, and $(G, \Sigma)$ be an $\mathbb{F}^{\times}$-labelled graph associated with $N$. We have $\left|M^{*}\right|=|M| \geq(1+\beta) r(M)=$ $(1+\beta)\left(\left|M^{*}\right|-r\left(M^{*}\right)\right)$, giving $\left|M^{*}\right| \leq\left(1+\beta^{-1}\right) r\left(M^{*}\right) \leq 2 \beta^{-1} r\left(M^{*}\right)$.

Now $k \leq r\left(M^{*}\right)-k \leq r(N) \leq|V(G)|+1 \leq 2|V(G)|$, so

$$
|E(G)|=|N|=\left|M^{*}\right| \leq 2 \beta^{-1}(|V(G)|+k) \leq 6 \beta^{-1}|V(G)| .
$$

Therefore $G$ has average degree at most $12 \beta^{-1}$. If $|V(G)|<2(k+1)$, then $2(k+1)-1 \geq r(N) \geq r\left(M^{*}\right)-k$ so $r\left(M^{*}\right) \leq 3 k+1$. This gives $\left|M^{*}\right| \leq 2 \beta^{-1}(3 k+1)<c$, a contradiction. Therefore $|V(G)| \geq 2(k+1)$, so there is a collection of $2(k+1)$ vertices of $G$ whose degrees sum to at most $12 \beta^{-1}(k+1)$, so there is a set $F \subseteq E(G)$ such that $|F| \leq$ $12 \beta^{-1}(k+1) \leq c$ and $r(N \backslash F) \leq r(N)+1-2(k+1)$ (since $A$ has at most $r(N)+1$ rows and $A[E(N)-F]$ has at least $2(k+1)$ zero rows). Now

$$
r\left(M^{*} \backslash F\right) \leq r(N \backslash F)+k \leq r(N)-2 k-1+k \leq r\left(M^{*}\right)-1 .
$$

Thus $F$ contains a cocircuit of $M^{*}$, which is a circuit of $M$, giving $g(M) \leq c$.

\section{The Main Result}

The following theorem implies Theorem 1.1 
Theorem 6.1. Let $\mathbb{F}$ be a finite field. If $\mathcal{M}$ is a minor-closed class of $\mathbb{F}$-represented matroids, then $\mathcal{M}$ is asymptotically good if and only if $\mathcal{M}$ contains all projective geometries over $\mathbb{F}_{\text {prime }}$.

Proof. If $\mathcal{M}$ contains all projective geometries over $\mathbb{F}_{\text {prime }}$, then $\mathcal{M}$

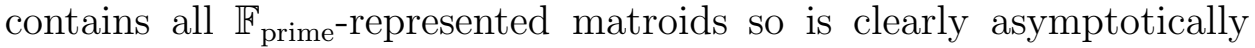
good. Suppose that $\mathcal{M}$ does not contain all projective geometries over $\mathbb{F}_{\text {prime }}$ but is asymptotically good. By Theorem 4.2 , there is an integer $k$ so that every vertically $k$-connected matroid $M \in \mathcal{M}$ satisfies $\operatorname{dist}(M, N) \leq k$ or $\operatorname{dist}\left(M^{*}, N\right) \leq k$ for some $\mathbb{F}$-represented frame matroid $N$; moreover, $N$ is vertically 3 -connected by Lemma 4.1, so by Corollary 2.4 we may assume the associated graph is connected. Let $\mathcal{M}_{k}$ denote the class of vertically $(2 k+3)$-connected matroids in $M$. Note that every such matroid is also vertically $k$-connected.

By Lemma 3.1, the class $\mathcal{M}_{k}$ is asymptotically good, so $\mathcal{M}_{k}$ contains an $(\alpha, \alpha)$-good sequence for some $\alpha \in(0,1)$. Let $\beta=(1-\alpha)^{-1}-1$ and let $c$ be the maximum of the two integers given by Lemmas 5.4 and 5.5 for $\mathbb{F}, \beta$ and $k$. Let $n_{0}$ be an integer so that $c \log n<\alpha n$ for all $n \geq n_{0}$.

There is a matroid $M \in \mathcal{M}_{k}$ so that $|M| \geq n_{0}, d(M)=g\left(M^{*}\right) \geq$ $\alpha|M|$ and $r(M) \geq \alpha|M|$. The last inequality gives $\left|M^{*}\right| \geq(1+$ $\beta) r\left(M^{*}\right)$, so by Lemma 5.4 or 5.5 we have $d(M) \leq c \log r\left(M^{*}\right) \leq$ $c \log \left|M^{*}\right|<\alpha\left|M^{*}\right|$, a contradiction.

\section{ACKNOWLEDGEMENTS}

We thank Navin Kashyap for his careful reading and very useful advice on the manuscript.

\section{REFERENCES}

[1] N. Alon, S. Hoory and N. Linial, The Moore bound for irregular graphs, Graphs Combin. 18 (2001), 53-57.

[2] N. Kashyap, A decomposition theory for binary linear codes, IEEE Transactions on Information Theory 54 (2008), 3035-3058.

[3] J. Geelen, B. Gerards and G. Whittle, The highly connected matroids in minor-closed classes, arXiv:1312.5012 [math.CO].

[4] J. G. Oxley, Matroid Theory (2nd edition), Oxford University Press, New York, 2011.

[5] N. Robertson and P. D. Seymour, Graph Minors. XVI. Excluding a non-planar graph, J. Combin. Theory Ser. B 89 (2003), 43-76. 